Trabajos Originales

\title{
CORRELACIÓN DE LA HISTEROSCOPIA Y BIOPSIA DIRIGIDA EN EL ESTUDIO DEL ENG ROSAMIENTO ENDOMEIRIAL PATOLÓGICO POR ULTRASONIDO
}

\author{
Macarena Socías T. ${ }^{1}$, Renato Vargas S. ${ }^{1}$, Alberto Costoya A. ${ }^{1}$, Bárbara Monje R. ${ }^{2}$, \\ María Teresa Haye M. ${ }^{3}$ \\ 1 Unidad de Endoscopia Ginecológica, Servicio de Obstetricia y Ginecología, Hospital San José, Universidad de \\ Santiago. ${ }^{2}$ Programa de Magíster en Bioestadística, Escuela de Salud Pública de la Universidad de Chile. ${ }^{3}$ Becada \\ Ginecología y Obstetricia, Hospital Clínico de la Universidad de Chile.
}

\begin{abstract}
RESUMEN
Objetivo: Correlacionar la histeroscopia con la biopsia dirigida en pacientes pre y postmenopáusicas con engrosamiento endometrial patológico al ultrasonido. Método: Estudio retrospectivo de 265 pacientes derivadas por engrosamiento endometrial patológico en la ecografía transvaginal y evaluadas mediante histeroscopia con biopsia dirigida. Resultados: $68,7 \%$ de las pacientes presentaron un aspecto histeroscópico benigno, $15,1 \%$ normal, $10,6 \%$ potencialmente maligno y 5,6\% maligno. Los diagnósticos anátomo patológicos más frecuentes fueron: pólipo endometrial $(n=92 ; 34,7 \%)$, endometrio proliferativo/ secretor $(n=84 ; 31,7 \%)$ y mioma submucoso $(n=38 ; 14,3 \%)$. Todos los cánceres endometriales $(n=9)$ se presentaron en mujeres postmenopáusicas sin TRH y con endometrio $\geq 11 \mathrm{~mm}$. La correlación entre histeroscopia y anatomía patológica para diagnóstico de patología benigna tuvo una sensibilidad y especificidad de $90,7 \%$ (95\% IC 85,2 - 94,3) y $65,4 \%$ (95\% IC 55,8 - 73,8); valor predictivo positivo y negativo fue de $80,2 \%(95 \%$ IC $73,8-85,4)$ y $82 \%(95 \%$ IC $72,3-88,7)$. Para diagnóstico de hiperplasia endometrial la sensibilidad y especificidad fue $60 \%$ (95\% IC $31,3-83,2)$ y $91,4 \%$ (95\% IC $87,3-94,2)$; el valor predictivo positivo y negativo fue de $21,4 \%$ (95\% IC $10,7-39,5)$ y $98,3 \%(95 \%$ IC $95,7-99,3)$. Para diagnóstico de cáncer endometrial la sensibilidad y especificidad fue de $95 \%(95 \%$ IC $65,5-99,5)$ y $97,9 \%$ (95\% IC 95,2 - 99); el valor predictivo positivo y negativo fue de $63,3 \%(95 \%$ IC $38,7-82,5)$ y 99,7\% (95\% IC 98,1 - 99,8). Conclusión: El $85 \%$ de las pacientes con engrosamiento endometrial presentaron una histeroscopia alterada (benigna, potencialmente maligna o maligna), confirmada en $80 \%$ de los casos por anatomía patológica. En un centro con experiencia la histeroscopia normal puede hacer innecesaria la biopsia. El aspecto potencialmente maligno a la histeroscopia puede ser un sobrediagnóstico, por lo que la anatomía patológica es imprescindible. Todos los cánceres endometriales fueron diagnosticados a la histeroscopia.
\end{abstract}

\section{PALABRAS CLAVE: Biopsia endometrial, cáncer endometrial, ecografía transvaginal, engrosamiento endometrial, histeroscopia}

\section{SUMMARY}

Objective: Comparison of hysteroscopy with biopsy in pre- and post -menopausic patients with endometrial thickening on ultrasound. Methods: Retrospective study of 265 patients with pathologic endometrial thickening on transvaginal ultrasound and evaluated with hysteroscopy and biopsy. Results: $68.7 \%$ of the patients had benign hysteroscopy results, $15.1 \%$ were normal, $10.6 \%$ were potentially malignant, and $5.6 \%$ were malignant. The most frequent biopsy results were: endometrial polyp $(n=92,34.7 \%)$, proliferate / 
secretor endometrium ( $n=84,31.7 \%)$, and submucosal myoma $(n=38,14.3 \%)$. All of the endometrial cancers $(n=9)$ were in post- menopausic women, with no history of hormone replacement therapy and with endometrial thickening $\geq 11 \mathrm{~mm}$. Sensitivity and specificity for hysteroscopy in comparison to biopsy for diagnosing benign pathology were $90.7 \%(95 \% \mathrm{Cl} 85.2$ - 94.3) and $65.4 \%(95 \% \mathrm{Cl} 55.8-73.8)$, and the positive and negative predictive values were $80.2 \%$ (95\% Cl $73.8-85.4)$ and $81.9 \%$ (95\% Cl $72.3-88.7$ ). Sensitivity and specificity for hysteroscopy in comparison to biopsy for diagnosing endometrial hyperplasia were $60 \%(95 \% \mathrm{Cl} 31.3-83.2)$ and $91.4 \%(95 \% \mathrm{Cl} 87.3$ - 94.2), the positive and negative predictive values were $21.4 \%(95 \% \mathrm{Cl} 10.7$ - 39.5) and $98.3 \%(95 \% \mathrm{Cl} 95.7$ - 99.3). Sensitivity and specificity for hysteroscopy in comparison to biopsy for diagnosing endometrial cancer were $95 \%(95 \% \mathrm{Cl} 65.5-99.5)$ and $97.9 \%$ $(95 \% \mathrm{Cl} 95.2$ - 99), positive and negative predictive values were $63.3 \%(95 \% \mathrm{Cl} 38.7-82.5)$ and $99.7 \%$ (95\% Cl 98.1 - 99.8). Conclusion: $85 \%$ of the patients with endometrial thickening had an abnormal hysteroscopy result, which latter were confirmed in $80 \%$ of the cases with biopsy. In a center with experience, a normal hysteroscopy result can make biopsy unnecessary. Potentially malignant hysteroscopys tend to be over diagnosed, making biopsy fundamental. All endometrial cancers were detected by hysteroscopy in our study.

\section{KEY WORDS: Endometrial biopsy, endometrial cancer, transvaginal ultrasound, endometrial thickening, hysteroscopy}

\section{INTRODUCCIÓN}

El grosor endometrial varía con el ciclo menstrual y la edad $(1,2)$. Los puntos de corte para considerar un grosor patológico del endometrio a la ecografía son $\geq 16 \mathrm{~mm}$ en edad reproductiva, $\geq 5 \mathrm{~mm}$ para la postmenopausia $\mathrm{y} \geq 8 \mathrm{~mm}$ en la postmenopausia con terapia de reemplazo hormonal (TRH). Algunos sostienen que el punto de corte no debe ser diferente para la mujer con TRH o sin ella $(3,4)$. En la práctica clínica, un grosor $\leq 4 \mathrm{~mm}$ es capaz de excluir patología significativa con un valor predictivo positivo (VPP) de $87,3 \%$ y un $90 \%$ de sensibilidad $(1,5,6)$. Con todo, el valor de la medición ecográfica del grosor endometrial parece ser un mejor predictor en la postmenopausia que en la premenopausia respecto a cáncer de endometrio $(7,8)$.

El engrosamiento endometrial requiere de otros métodos para un diagnóstico definitivo. Entre ellos, la histeroscopia, y recientemente la histerosonografía, son considerados procedimientos de primera línea, tanto en pacientes sintomáticas como asintomáticas $(9,10)$.

Independiente del grupo etáreo, un sangrado uterino anormal se debe a patología anatómica benigna en un 30 a $50 \%$ de los casos; en la mujer menor de 50 años se diagnostica una patología maligna en menos del $1 \%$, mientras sobre esta edad la cifra sube a $10-15 \%(1,9)$.

La utilidad de la histeroscopia ambulatoria para diagnosticar patología difusa o focalizada, y principalmente cáncer de endometrio ha sido reportada como un método altamente preciso por diversos autores $(7,11)$.
El objetivo de este trabajo es correlacionar la histeroscopia ambulatoria y la biopsia endometrial dirigida en el estudio del engrosamiento endometrial.

\section{MATERIAL Y MÉTODOS}

Desde diciembre de 2004 hasta marzo de 2006 la Unidad de Endoscopia Ginecológica del Servicio de Obstetricia y Ginecología del Hospital San José realizó un total de 905 histeroscopias, de las cuales 265 cumplieron los criterios de inclusión de este estudio que fueron:

- Pacientes derivadas por presentar endometrio patológico al ultrasonido transvaginal, tanto en edad reproductiva como en postmenopausia. Se consideraron patológicos aquellos grosores endometriales $\geq 16 \mathrm{~mm}$ en edad reproductiva, $\geq 5 \mathrm{~mm}$ en la postmenopausia $\mathrm{y} \geq 8 \mathrm{~mm}$ en la postmenopausia con terapia de reemplazo hormonal (TRH).

- Histeroscopias ambulatorias calificadas como técnicamente satisfactorias.

- Biopsia dirigida por histeroscopia.

Se consignó la edad, historia obstétrica, patología ginecológica asociada, uso de anticonceptivos, TRH y presencia de metrorragia.

Todas las pacientes fueron sometidas a una histeroscopia ambulatoria con medio gaseoso y biopsia dirigida, a través de un histeroscopio Hopkins II (Karl Storz) de $4 \mathrm{~mm}$ de diámetro, visión de $30^{\circ}$ y biótomo de $7 \mathrm{Fr}$ para biopsia parahisteroscópica. Todas las intervenciones fueron realizadas indistintamente por dos miembros del equipo. Las histeroscopias fueron indicadas en fase proliferativa precoz, y las pacientes se premedicaron 
con analgésico y antiespasmódico al menos una hora antes del procedimiento; en los casos de permeabilidad cervical dificultosa se administraron además $200 \mathrm{mg}$ de misoprostol por vía vaginal la noche previa al procedimiento.

Según la evaluación histeroscópica, el aspecto endometrial en todas las pacientes se clasificó como: normal, benigno, potencialmente maligno y maligno. Se consideraron patologías benignas los pólipos endometriales, miomas submucosos, endometritis, sinequias, tabiques, metaplasia endometrial y restos ovulares. Como potencialmente malignos las hiperplasias endometriales, y malignos a los adenocarcinomas. La anatomía patológica fue clasificada igualmente como: normal, patología benigna, patología potencialmente maligna y patología maligna.

La información obtenida fue tabulada en una base de datos Excel y analizada con STATA 7,0 software, utilizando test de Kruskal-Wallis para los datos ordinales, test de t Student para los datos continuos y test de Fisher para los datos categóricos.

\section{RESULTADOS}

Las pacientes tuvieron una media de edad de $50,9 \pm 10,2$ años (rango: $26-86$ ). Un 49\% fueron postmenopáusicas; $5,3 \%$ de estas últimas eran usuarias de $\mathrm{TRH}$. El $62 \%$ de la muestra total presentó antecedentes de metrorragias.
La visión histeroscópica fue calificada como aspecto benigno en 182 casos (68,7\%), normal en 40 casos $(15,1 \%)$, potencialmente maligno en 28 casos $(10,6 \%)$ y maligno en 15 casos $(5,6 \%)$. Los diagnósticos específicos más frecuentes a la histeroscopia fueron: pólipo endometrial en 94 casos $(35,5 \%)$, mioma submucoso en 44 casos $(16,6 \%)$, normal en 40 casos (15\%), sugerente de hiperplasia endometrial en 28 casos (11\%), y sospecha de cáncer en 15 casos (5,6\%) (Figura 1).

Los diagnósticos anátomo patológicos más frecuentes fueron: pólipos endometriales en 92 casos $(34,7 \%)$, endometrio proliferativo/secretor en 84 casos $(31,7 \%)$, miomas submucosos en 38 casos $(14,3 \%)$, atrofia endometrial en 20 casos $(7,6 \%)$, hiperplasias endometriales en 10 casos $(3,8 \%)$ y adenocarcinoma endometrioide en 9 casos $(3,4 \%)$ (Figura 2$)$.

Cuando la histeroscopia fue normal $(n=40)$, las biopsias fueron también diagnosticadas como normales en todos los casos, otorgando un valor predictivo positivo (VPP) de 100\% (Test de Fisher $p$ $<0,0001$ ).

Las histeroscopias clasificadas como benignas $(\mathrm{n}=182)$ resultaron ser normales a la anatomía patológica en 36 casos (20\%), y confirmaron una patología benigna en 146 casos (80\%). En ningún caso en este grupo se encontró patología potencialmente maligna o patología maligna a la biopsia. Para patología benigna el VPP fue de $80,2 \%$ (95\% IC 73,8 - 85,4); valor predictivo negativo (VPN) de

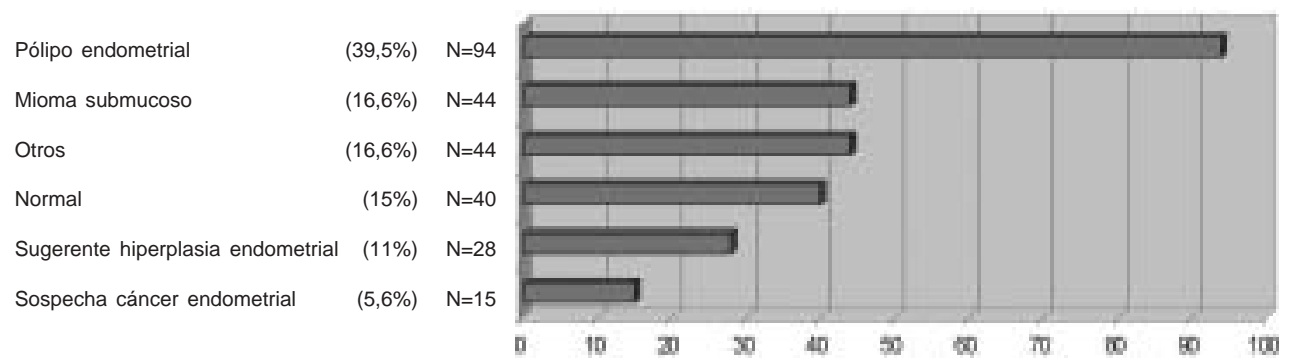

Figura 1. Diagnósticos específicos a la histeroscopia.

Pólipo endometrial
Endometrio proliferativo normal
Mioma submucoso
Atrofia endometrial
Endometrio secretor normal
Otros
Hiperplasia endometrial
Adenocarcinoma endometrial

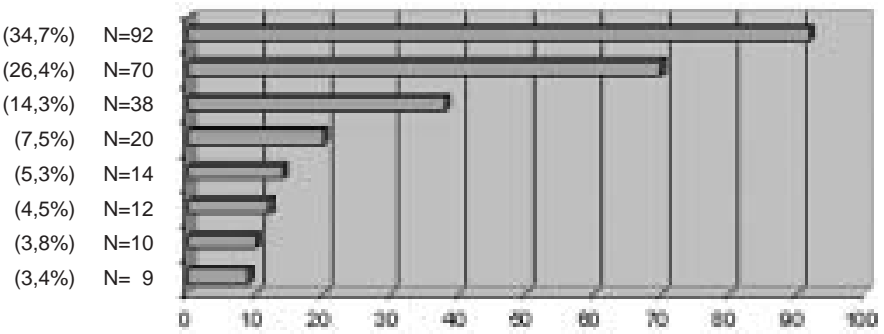

Figura 2. Diagnósticos anatomopatológicos. 
$82 \%$ (95\% IC 72,3 - 88,7); sensibilidad (S) de 90,7\% $(95 \%$ IC 85,2 - 94,3) y especificidad (E) de $65,4 \%$ (95\% IC 55,8 - 73,8).

Al analizar las histeroscopias potencialmente malignas $(n=28)$, se encontraron $6(21 \%)$ hiperplasias de endometrio a la biopsia, 4 (14,3\%) endometrios proliferativos normales, $4(14,3 \%)$ endometrios secretores normales, 9 (32\%) pólipos endometriales y $5(18 \%)$ endometritis. En este grupo, la impresión diagnóstica endoscópica para hiperplasia endometrial tuvo un VPP de $21,4 \%$ (95\% IC 10,7 - 39,5); VPN de 98,3\% (95\% IC 95,7 - 99,3); S de $60 \%(95 \%$ IC $31,3-83,2)$ y E de $91,4 \%$ (95\% IC 87,3-94,2).

En el grupo de las histeroscopias de aspecto maligno $(n=15)$, se diagnosticaron por anatomía patológica $9(60 \%)$ adenocarcinomas endometriales, $4(27 \%)$ hiperplasias de endometrio y $2(13 \%)$ pólipos endometriales. La histeroscopia de aspecto maligno para el diagnóstico de adenocarcinoma de endometrio tuvo un VPP de 63,3\% (95\% IC 38,7 82,5); VPN de $99,7 \%$ (95\% IC 98,1 - 99,8); $\mathrm{S}$ de $95 \%(95 \%$ IC $65,5-99,5)$ y E de $97,9 \%(95 \%$ IC 95,2 - 99,5).

Todos los cánceres endometriales confirmados por anatomía patológica se presentaron en pacientes postmenopáusicas con una media de edad de 61,5 años (95\% IC 51 - 71) vs 50,5 años (95\% IC 49 - 51) en pacientes sin cáncer (t Student $p<$ 0,0014). Ninguna de las pacientes con cáncer endometrial confirmado por anatomía patológica era usuaria de TRH (Test de Fisher $p>0,39$ ).

El grosor endometrial promedio de la muestra estudiada fue 15,7 $\pm 6,3 \mathrm{~mm}$. En las mujeres con diagnóstico de cáncer endometrial a la anatomía patológica, el grosor endometrial promedio fue de $18,4 \pm 6,3 \mathrm{~mm}$ vs $15,6 \pm 6,3 \mathrm{~mm}$ en las mujeres sin cáncer, diferencia que no fue estadísticamente significativa (t Student $p>0,19$ ). En la Tabla I se resumen la relación entre patología diagnosticada por anatomía patológica, grosor endometrial y variables epidemiológicas.

No se encontraron cánceres con grosor endometrial menor a $11 \mathrm{~mm}$. La distribución según grosor endometrial de los 9 cánceres en nuestra muestra fue: 4 con grosor entre 9 y $15 \mathrm{~mm}, 3$ con grosor entre 16 y $24 \mathrm{~mm}$ y 2 con grosor $\geq 25 \mathrm{~mm}$, diferencia no significativa (Test de Kruskal-Wallis $p$ $>0,24)$.

El antecedente de metrorragia en las pacientes con cáncer endometrial tuvo una incidencia de 0,66 $(95 \%$ IC $0,28-1,051)$ vs $0,61(95 \%$ IC $0,5-0,6)$ en pacientes sin cáncer, diferencia que no fue estadísticamente significativa (Test de Fisher $p>0,53$ ).

\section{DISCUSIÓN}

Las pacientes estudiadas fueron predominantemente peri y post-menopáusicas, de las que un bajo porcentaje eran usuarias de TRH. La edad en las mujeres con cáncer endometrial fue significativamente mayor en comparación con las mujeres sin cáncer $(p<0,0014)$. La influencia de la TRH en esta patología no tuvo asociación estadísticamente significativa $(p>0,39)$, lo cual podría deberse al escaso número de mujeres usuarias de TRH $(n=7)$ en nuestro estudio.

La presencia de metrorragia no tuvo relación

Tabla I

\section{DIAGNÓSTICO HISTOPATOLÓGICO, HALLAZGOS EPIDEMIOLÓGICOS Y GROSOR ENDOMETRIAL}

\begin{tabular}{lcccccc}
\hline $\begin{array}{l}\text { Diagnóstico } \\
\text { histopatológico }\end{array}$ & $n$ & $\begin{array}{c}\text { Frecuencia } \\
(\%)\end{array}$ & $\begin{array}{c}\text { Edad } \\
\text { (años, pro- } \\
\text { medio } \pm D S)\end{array}$ & $\begin{array}{c}\text { Pre meno- } \\
\text { páusica(n) }\end{array}$ & $\begin{array}{c}\text { Post meno- } \\
\text { páusica }(n)\end{array}$ & $\begin{array}{c}\text { Grosor endometrial } \\
\text { (mm, prome- } \\
\text { dio } \pm S \text { ) }\end{array}$ \\
\hline $\begin{array}{l}\text { Mioma submucoso } \\
\text { Endometrio prolifera- }\end{array}$ & 38 & 14,3 & $52,4 \pm 10,5$ & 21 & 17 & $17,8 \pm 7,05$ \\
$\quad$ tivo/secretor & 84 & 31,7 & $47,8 \pm 8,1$ & 54 & 30 & $14,5 \pm 5,30$ \\
Pólipo endometrial & 92 & 34,7 & $50,9 \pm 9,2$ & 44 & 48 & $16,8 \pm 6,58$ \\
Hiperplasia endometrial & 10 & 3,7 & $46,6 \pm 10,1$ & 7 & 3 & $15,9 \pm 4,31$ \\
Atrofia endometrial & 20 & 7,5 & $62,6 \pm 9,6$ & 1 & 19 & $5,1 \pm 2,40$ \\
Cáncer endometrial & 9 & 3,4 & $61,5 \pm 13$ & 0 & 9 & $18,4 \pm 6,35$ \\
Restos ovulares & 3 & 1,1 & $37,6 \pm 10,7$ & 3 & 0 & $16,7 \pm 0,58$ \\
Metaplasia ósea & 3 & 1,1 & $47 \pm 5$ & 2 & 2 & $17,6 \pm 8,50$ \\
Endometritis & 6 & 2,2 & $43,4 \pm 9,7$ & 3 & 121 & $13 \pm 4,12$ \\
\hline TOTAL & 265 & 100 & $50 \pm 10,2$ & 135 & $15,7 \pm 6,30$ \\
\hline
\end{tabular}


estadísticamente significativa para patología endometrial maligna $(p>0,53)$, presentándose en la misma proporción en las demás patologías evaluadas.

En nuestra experiencia, un grosor endometrial anormal significa encontrar algún tipo de patología a la histeroscopia en un $85 \%$ de los casos, confirmando el valor de la medición ecográfica del grosor endometrial $(3,12)$. El hecho de que en las 40 histeroscopias clasificadas como normales no se encontrara ninguna anormalidad a la anatomía patológica confirma que cuando en un centro con experiencia histeroscópica la cavidad uterina es calificada como normal, podría no ser necesario tomar una biopsia, lo que debiera ser confirmado con un número mayor de pacientes.

EI VPP de la histeroscopia para diagnosticar benignidad fue del $100 \%$ si se consideran las anatomías patológicas normales y las de patología benigna. Para esta última, el VPP fue de $80,2 \%$, confirmando el alto valor que tiene la histeroscopia para predecir benignidad.

En cuanto a la predicción del grupo histeroscópico calificado como potencialmente maligno $(n=28)$, se encontró el menor VPP $(21,4 \%)$, lo que significa que esta impresión histeroscópica tiene una escasa relación con hiperplasia endometrial, siendo su mayor utilidad en la práctica el descartarlas, al tener un VPN de $98,3 \%$ y $E$ de $91,4 \%$. Cabe destacar que los principales diagnósticos confundentes en esta categoría corresponden a las endometritis crónicas, endometrios secretores en histeroscopias realizadas en períodos inadecuados por error en citación de la paciente y estimulación estrogénica prolongada, todos factores a tener en cuenta $(1,5)$.

En los casos en que la histeroscopia fue calificada como maligna $(n=15)$ se concentró la totalidad de los 9 cánceres endometriales encontrados en las 265 pacientes de este estudio. Esto significa que la histeroscopia es altamente eficaz en detectar un cáncer endometrial y altamente específica para descartar la "normalidad" del endometrio.

En las pacientes con grosor endometrial $<11$ $\mathrm{mm}$ no se encontraron histeroscopias de aspecto maligno como tampoco biopsias que indicaran patología maligna. Para cáncer endometrial no hay significancia estadística directa en relación al grosor del endometrio $(p>0,19)$. La falta de relación entre grosor endometrial y patología maligna, así como la asociación con metrorragia, puede ser debida también al tamaño muestral, ya que otros autores $(7,13)$ han demostrado una clara asociación de estas dos variables con el cáncer endometrial.
En resumen, este trabajo confirma que la histeroscopia ambulatoria es de gran utilidad en la evaluación de aquellos endometrios engrosados a la medición por ultrasonografía. En primer lugar, el sólo hecho de encontrar esta alteración significa la confirmación de algún tipo de patología: benigna, potencialmente maligna o maligna a la visión endoscópica en el $85 \%$ de los casos en nuestra experiencia. A su vez en un $80 \%$ este diagnóstico histeroscópico significa algún diagnóstico anátomo patológico de anormalidad, confirmando a su vez el valor predicativo de la histeroscopia en relación al diagnóstico final anátomo patológico. En segundo lugar, en un centro con experiencia en histeroscopia, el encontrar una cavidad uterina definida como normal podría hacer innecesaria la toma de biopsia. En tercer lugar, el grupo de histeroscopias clasificadas como malignas concentra el $100 \%$ de los cánceres de endometrio, y aunque en este grupo existen también otras patologías, ningún cáncer se escapó al diagnóstico endoscópico. En cuarto lugar, y a pesar de lo anterior, las endoscopias calificadas como potencialmente malignas pueden significar un sobrediagnóstico que incluye una variedad de patologías endometriales, pero habitualmente benigna siendo imprescindible el diagnóstico específico anatomopatológico.

\section{BIBLIOGRAFÍA}

1. Kairo B, Berga S. Bleeding problems in midlife. En: Gynecology and Obstetrics CD-ROM 2004 Edition. Lippincott Williams \& Wilkins, V5, Chap 23.

2. Gormaz G, Prado S, Duque G, et al. Caracterización ultrasonográfica del endometrio durante el ciclo menstrual ovulatorio espontáneo. Rev Chil Obstet Ginecol 1992;57(4):257-62.

3. Epstein E, Valentin L. Managing women with postmenopausal bleeding. Best Pract Res Clin Obstet Gynaecol 2004;18:125-43.

4. Smith-Bindman R, Kerlikowske K, Feldstein VA, et al. Endovaginal ultrasound to exclude endometrial cancer and other endometrial abnormalities. JAMA 1998; 280:1510-7.

5. Goldstein S. Menorrhagia and abnormal bleeding before the menopause. Best Pract Res Clin Obstet Gynaecol 2004;18:59-69.

6. Jain A, Santoro N. Endocrine mechanisms and management for abnormal bleeding due to perimenopause changes. Clin Obstet Gynecol 2005;2:295-311.

7. Hatasaka $\mathrm{H}$. The evaluation of abnormal uterine bleeding. Clin Obstet Gynecol 2005;2:258-73.

8. Hammond R, Johnson J. Endometrial hyperplasia. Curr Opin Obstet Gynecol 2004;14:99-103. 
9. Bettocchi S, Nappi L, Ceci O, et al. Hysteroscopy and menopause: past and future. Curr Opin Obstet Gynecol 2005;17:366-75.

10. O'Conell LP, Fries MH, Zerinque E, Brehm W. Triage of abnormal postmenopausal bleeding: A comparison of endometrial biopsy and transvaginal sonohysterography versus fractional curettage with hysteroscopy. Am J Obstet Gynecol 1998;178:956-61.

11. Vargas R, Walton R, Morales F. Accuracy of outpatient hysteroscopy in the diagnosis of endometrial cancer.
Department of Gynaecological Endoscopy, San José Hospital. Santiago, Chile. FIGO, November 2003.

12. Litta P, Marlin F, Saccardi C, Pozzan C, et al. Role of hysteroscopy with endometrial biopsy to rule out endometrial cancer in postmenopausal women with abnormal uterine bleeding. Maturitas 2005;50:117-23.

13. Fleischer AC, Wheeler JE, Lindsay I, et al. An assessment of the value of ultrasonographic screening for endometrial disease in postmenopausal women without symptoms. Am J Obstet Gynecol 2001;184:70-5. 